\title{
ERRATUM
}

Chai-Fong Lee $\cdot$ Hsin-Yi Pu $\cdot$ Lian-Chin Wang

Ronald J. Sayler $\cdot$ Ching-Hui Yeh $\cdot$ Shaw-Jye Wu

\section{Mutation in a homolog of yeast Vps53p accounts for the heat and osmotic hypersensitive phenotypes in Arabidopsis hit1-1 mutant}

Published online: 14 February 2006

(C) Springer-Verlag 2006

\section{Planta (2005) DOl: 10.1007/s00425-005-0216-6}

Unfortunately, Fig. 2 was published with errors. The correct figure is given here:

The online version of the original article can be found at http:// dx.doi.org/10.1007/s00425-005-0216-6

C.-F. Lee · H.-Y. Pu · L.-C. Wang · C.-H. Yeh · S.-J. Wu ( $ه)$ Department of Life Sciences, National Central University, 300 Jhong-da Road, 320 Jhong-li City, Taoyuan, Taiwan E-mail: jyewu@cc.ncu.edu.tw

Tel.: + 886-3-4227971

Fax: + 886-3-4228482

R. J. Sayler

Department of Plant Pathology, University of Arkansas, 217 Plant Science, Fayetteville, AR 72701, USA 


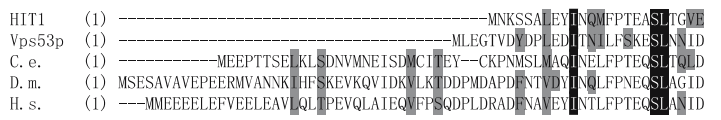

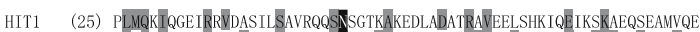
Vps53p (29) ELISITRSYKKQLQEDILKEE---NELKEHPKNSAEIEASLRKVFRDFKETQDVSASEELTISN C. e. (53) SIIASVEGEIGELDNELAYLETNANVSERGEEALKHAQDAMIELEKSIGSIRERTKSSDEIVR D. m.
H. s. (63) EVVNKIRLKIRRLDDNIRTVVRGQTNVGQDGRQALEEAQKAIQQLFGKIKDIKDKAEKSEQMVKE

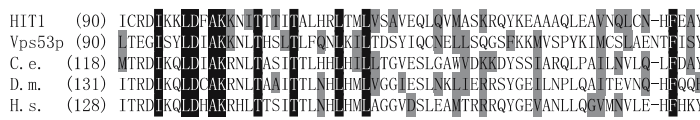
HIT1 (154) RDVPKITELREKLNVIKQILKSHVFFSFFSLGTGKETEETN---LLQQKLSDSCLVVDALËPSVRE
Vps53p (155) KSLDEINYLLSISRL KGDTLSKIKQNYNALFSGGNISBHD-TALTMELREGACELLDCDTSTRA C. e. (182) KESDQTANLSGQLDKL KASLTIQLAKDLKNAFQTGQLSDR-------ITDMCRVAAALEGGVK D. m.
H. s. (192) MGIPQIRQLSERVKAAQTELGQILADFEEAFPSQGTKRP—GGPSNLRDACLVANILDPRIKK

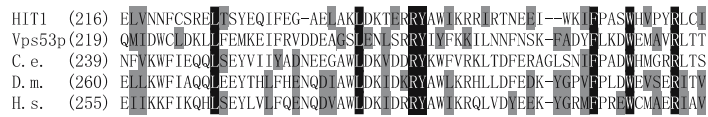

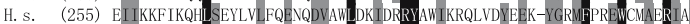

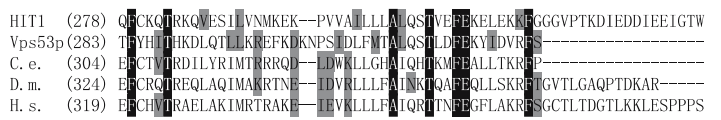

HIT1 (341) EDNSQNISKIRKKYEKKFAASQETEENGFQQEKTGNKDLSVTGAGFNFRGMISSCFEPHITPYIF
Vps53p (330) -

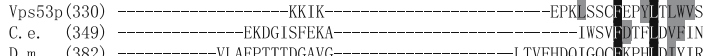

H. $\mathrm{s}$. (382) TNPFLEDEPTPEMEELATEKGDLDQPK---------KPKAPDNPFHGIVSKCHEPHU YVYIE

HIT1 (406) LEEEKTLMDDLEKIVQEESWD-----VEDGSQNNVLSSSTQLFSNIKKSLKRCNTLSKN----QT

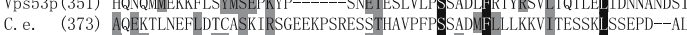

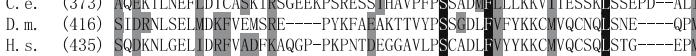

HIT1 (462) FNLFKVFQRVLKAYITTKLFFKLP---------KGGTGIVAAATGMDG--

Vps53p (410) TSLANFFSRWLQTYSQKILLPLLLPDNIEVQDKLEAAKYTVLLINTADYCAT

C. e. (436) RDVIGVVRVCLRGYATSCLVAFLP---SLGSQQSGAANLFSL IREEIAYP------_----

D. m. (473) YDLALVFKKYLREYASKVLEGSTPKLVPATTSSSIGKSVSLLTRDMQNLSTAAGQVFHNFLKEGD

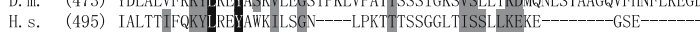

HIT1 (500) QIKVSERDERVICYIVNSAEYCHKTSGELAENVSEIIDPHYADGVDMSEVQDEFSAVITKALVTL Vps53p (462) TIDQLEDKLSEFSGNREKLAVSFTK KNIIYDDLLAKGTSFLLENRVIPLDLNFVWREFINVDWSNA C. e. (483) -R-LTPDQQFLVCCILATADICAETSIQLQEKLSQRIPG-----VDISQETEAFYSITNQSLQVL D. m. (538) TQRFARDDLVRICCVLTTGEYCLETVQQLEDKLKEKVTSAYVSKIDMSEEKDVFHRI ISVCIQLL

HIT1 (565) VLGLETKFDTEMAVMTRVP---------WSTLESVGDQSGYVNGINTVLSGSIPVLGKLLTPVps53p (527) AIEDYSRYMVTLKSVLKMP-------ALTDASIKQQQEQPSTLAFILSQFNRDVYKWNFLDKVID C. e. (541) VQDVESTCDAMLQSISKVKCSIFVRIEKTWTAVDCVGDESPFIGSMRAHLRQAVPLIRDMLSDR D. m. (603) VQDLEAGCEASLQAMAKVQ---------WQHINNVGDQSAFISSLCGNFKQTVPTIRDTLASS H. s. (606) VQDLDAACDPALTAMSKMQ--------WQNVEHVGDQSPYVTSVILHIKQNVPIIRDNLASTR

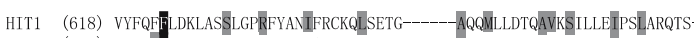
Vps53p (585) IITTNFVSNTIRLLQPVPPFSLAGSKRKFETR-TVVNIGEQLLLDLELLKEIFHTLPESVSNDSD C. e. (606) KYFAHFCLKLATQLAHKFVGSLFRCRTISTHG----AEQLLLLETHSLKTFLLSVPSIDSIINS D. m. (658) KYFTQFCHRFVAAFIPKFI NVLYRCKLTLSDGSNNVLGCEQLLLDTHSLKTALLELPSVGSSVN

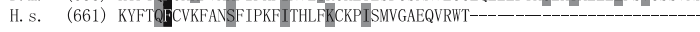

HIT1 (676) -----TAASYSKFEVSREMSRAEALLLKVILS--P-IDSVADTTYRALFP-EGTPMEFQRILEIKGGLK Vps53p (649) LRENTSYKRVKRHADNVIDQLLKFIKLLMAPLDSADDYYETYSKLTNNNPDSAVWSFVLALKGI C. e. (665) K----PPTAYVTSVNAALTKAEMIL KVVMCSLETVDEFVEQY IKLLP-ASDAAEMQKVLEMKGVK D. m. (723) K----APTSYTKVVVKDMTRAEMI IKVVMTPVQPPAHFTQQVLKLLP-DITIAEYQKILDMKAVK

H. s. $\quad(700)$

HIT1 (732) KAD̄QQSILDDFNKHGPĞGTQQSWAAAMPQPMPTPPAPPLAITN--PATAAGF IANSEDVLTRAAA Vps53p (714) WDLALWKKLWSAYNLETDDTDEGSRPDSNRDLF IFKWDKVLLGQFENNLARMQDPNWSKFVRQD C. e. (725) RQEHSAVLNAYRLKIGASGSDP IQQ------------_-- SNSLISRIGGALPTVGSAL

$\begin{array}{ll}\text { D. m. } & (783) \text { RVDQLQLIDLFKHTASAAAVSGLIEPTTGEEETQGAETVVATSGTTDDAETSAVPTETTTATSS } \\ \text { H. s. }\end{array}$

HIT1 (795) LGRGAASTGFKKF IALTEAAKDRKDGPLRRLFNAS--------

Vps53p (779) KISPPVMKRIVSTPQIQQQKEEQKKQSLSVKDFV SHSRFFNRGT

C. e. (770) VSEAFNAVVSMAADGLSDQAVTSSIDKLKRFERLVKRQL-----
D. m. (848) PKRAFIFSVGSFTGSADKNADGSSQTGIRKLENLLKKRFP----

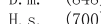

Fig. 2 Amino acid sequence comparison of HIT1 protein with their putative orthologs in C. cerevisiae (Vps53p, accession number P47061), C. elegans (C.e., accession number CAA81595), D. melanogaster (D.m., accession number AAF51022) and H. sapiens (H.s., accession number AAS20944). Sequence alignment was performed with Vector NTI software (Invitrogen, Carlsbad, CA, USA). Amino acids which share identity (black-shaded) and similarity (gray-shaded) are indicated 